\title{
EFEITOS DA CRISE ECONÓMICA E DAS POLÍTICAS DE AUSTERIDADE NA SAÚDE E NO ACESSO AOS CUIDADOS DE SAÚDE DA POPULAÇÃO MIGRANTE EM PAÍSES DO SUL DA EUROPA: REVISÃO SCOPING
}

\author{
Effects of the economic crisis and austerity policies on health and \\ health care access of migrant population in southern European \\ countries: scoping review
}

\author{
Nuno Moita Jordão* \\ Cláudia de Freitas** \\ Manuel García Ramírez ${ }^{* * *}$
}

\begin{abstract}
Resumo. O objectivo deste artigo é reunir e sintetizar o conhecimento existente sobre o impacto da crise económica e das consequentes políticas de austeridade no estado de saúde e no acesso aos cuidados de saúde das populações migrantes em países do sul da Europa, nomeadamente Portugal, Espanha, Itália e Grécia. Os resultados analisados indicam que a crise económica e as políticas de austeridade tiveram um impacto negativo na saúde e no acesso aos cuidados de saúde dos migrantes em três países, já que não se encontraram dados relativos a Portugal. Os seus efeitos negativos espelham-se na saúde mental, saúde ocupacional, doenças transmissíveis e não transmissíveis, saúde infantil e na perceção subjetiva de saúde. A acessibilidade dos cuidados de saúde tornou-se mais limitada em Espanha, especialmente para a população de imigrantes em situação irregular.
\end{abstract}

Palavras-chave: crise económica; políticas de austeridade; estado de saúde; acesso aos serviços de saúde; migrantes.

Abstract. The aim of this article is to gather and synthesize existing
knowledge about the impact of the economic crisis and the consequent
austerity policies on health condition and health care access of migrant
populations in southern European countries, namely Portugal, Spain, Italy
and Greece. The results analyzed indicate that the economic crisis and the
austerity policies had a negative impact on health and health care access
of migrants in three countries, since no data were found about Portugal.
The negative effects are reflected in mental health, occupational health,

${ }^{*}$ Centro Hospitalar Universitário do Algarve. Faro, Portugal.

${ }^{* *}$ Instituto de Saúde Pública da Universidade do Porto. Porto, Portugal.

${ }^{* * *}$ Universidade de Sevilha. Sevilha, Espanha. 
communicable and noncommunicable diseases, child health and in the subjective perception of health. Health care accessibility has become more limited in Spain, especially for irregular immigrants population.

Keywords: economic crisis; austerity policies; health condition; health care access; migrants.

\section{Introdução}

A saúde da população migrante nos países do Sul da Europa é um tema que requer a máxima atenção. A chegada massiva de imigrantes, muitas vezes em condições extremas, aos países do Mediterrâneo exige que seja avaliada a capacidade dos seus sistemas de saúde para dar resposta às necessidades destas pessoas. Apesar da natureza dos atuais fluxos migratórios diferir consideravelmente dos fluxos anteriores, este conhecimento poderá permitir antecipar as fortalezas e debilidades destes países fronteiriços com respeito a esta tarefa.

Os países do sul da Europa foram severamente afetados pela crise económica (Márquez, Urraza, 2016) de 2008, à qual responderam com a aplicação de diferentes políticas de austeridade. Não obstante, os setores da saúde, educação e segurança social foram os que sofreram mais cortes (Karanikolos, 2016).

Embora muitos considerem a imigração um direito humano, este fenómeno é intensamente político sendo mais ou menos aceite consoante os países de acolhimento.

Atitudes negativas perante a imigração podem ter um impacto igualmente negativo na saúde das populações migrantes, expondo-as a iniquidades que colocam em causa a sua integração (Heras-Mosteiro, Sanz-Barbero, OteroGarcia, 2016; Ingleby et alii, 2005).

Quando comparadas com as populações nativas, as populações migrantes tendem a apresentar "menor poder político, económico e social" (Malmusi, Ortiz-Barreda, 2014, p. 688), o que coloca em causa a igualdade de acesso às oportunidades e recursos disponíveis nos países de acolhimento, observando-se situações de injustiça social que podem ter um impacto negativo na saúde (García-Ramírez, Balcázar, De Freitas, 2014). O atenuar do chamado 'efeito do imigrante saudável' com o passar dos anos, segundo o qual os imigrantes apresentam melhores indicadores de saúde do que a população autóctone à chegada, será em parte explicado pela existência destas iniquidades (Malmusi, 2015).

No entanto, os processos migratórios continuam a representar um desafio para os princípios, valores e políticas das comunidades internacionais e países receptores ao terem "de enfrentar as necessidades de populações mais diversas" (Vázquez et alii, 2016, p. 396). 
Passada quase uma década do início da crise económica torna-se relevante fazer este estudo, de maneira a monitorizar o balanço do impacto da crise e das políticas de austeridade na saúde e no acesso aos cuidados da população migrante em Portugal, Espanha, Itália e Grécia, uma vez que, até ao momento, não foram ainda realizados estudos que comparem a evidência gerada nesses países. A escolha destes países deve-se pelo facto de todos eles se localizarem no sul da Europa e de terem sofrido fortes consequências decorrentes da crise económica global, o que gerou acentuados constrangimentos nos seus sistemas de saúde.

As alterações efetuadas nas políticas de saúde divergiram nos vários países em estudo. Em Portugal e na Grécia, além da redução dos orçamentos destinados ao setor da saúde e da centralização de serviços, foram aumentadas as taxas moderadoras que condicionam o acesso aos serviços de saúde, e muito embora seja oferecida isenção aos grupos com menos possibilidades económicas isso nem sempre é operacionalizado na prática (Legido-Quigley et alii, 2016). No entanto, foi em Espanha que se produziram as alterações mais marcantes, através da aplicação do Real Decreto-lei 16/2012 que restringe o acesso aos serviços de saúde a imigrantes indocumentados, salvo em casos de urgência, gravidez ou menores de idade (Boletín Oficial del Estado, 2012).

A organização centralizada dos organismos públicos portugueses e gregos contrasta com as políticas descentralizadas de Espanha e Itália, geridas pelas diversas comunidades autónomas regionais. Neste âmbito, o gozo de independência legislativa permite a aplicação de diferentes respostas pelas comunidades que têm partidos políticos ou ideologias opostas às do governo central, como é o caso da Catalunha ou Andaluzia em Espanha (Castano et alii, 2016).

A severidade dos cortes foi diferente em todos os países. Portugal e Grécia foram sujeitos a uma intervenção externa da Troika (composta pelo Fundo Monetário Internacional, Banco Central Europeu e Comissão Europeia) para controle da despesa pública, através da implementação de políticas de austeridade (Legido-Quigley et alii, 2016). Por outro lado, Espanha e Itália ao partilharem políticas regionais fortes não assinaram nenhum memorando, mas tiveram uma introdução de medidas pontuais e de autorregulação.

Estes países partilham um aspeto determinante no contexto das migrações que é a fronteira Sul e Sudeste da Europa, consideradas actualmente como as portas de entrada e de trânsito mais importantes. Assim sendo, é inequívoca a necessidade de avaliar o grau de preparação para atender e gerir a saúde destes novos fluxos migratórios de largas dimensões, considerando que têm de conviver com as populações migrantes previamente instaladas.

É também relevante porque todos os países têm um papel diferente na receção de novos imigrantes e permitem antecipar distintos aspetos. Dadas as 
suas localizações geográficas, a Grécia é, de todos eles, o mais débil e o que recebe maior pressão migratória procedente da Turquia, ao contrário da Itália e da Espanha, que têm como portas de entrada a Líbia e Marrocos, respetivamente, com migrantes provenientes, em qualquer dos casos, do Médio Oriente e do Continente Africano (International Organization for Migration, 2015). Portugal é, provavelmente, o país que menos sofre esta pressão migratória e onde as principais populações migrantes provêm das antigas colónias.

Por outro lado, os resultados obtidos são importantes medidores do nível de sensibilidade, competência e equidade dos sistemas de saúde e dos seus profissionais. Neste âmbito, constata-se que a União Europeia está muito focada na política de saúde migratória, ao investir em ferramentas de análise de dados, tal como o Índice das Políticas de Integração dos Imigrantes (MIPEX), ou em programas que colmatem a acessibilidade e equidade nos serviços de saúde.

\begin{abstract}
A adopção de um foco integral com respeito ao direito à saúde requer que os planos, as políticas e as estratégias nacionais prevejam as necessidades em saúde e a vulnerabilidade da população imigrante. Tais intervenções têm de considerar as iniquidades sanitárias, os obstáculos do acesso à saúde e outros fatores em saúde que afetam a saúde dos imigrantes, como os determinantes sociais. (Organización Internacional para las Migraciones, 2013, p. 56)
\end{abstract}

Indirectamente, estes resultados também são valiosos porque permitem-nos refletir sobre o impacto dos recentes aumentos de movimentos políticos extremistas, que incitam ao racismo e à xenofobia populistas, acentuando a vulnerabilidade de populações imigrantes em relação aos seus direitos, tais como a saúde e o acesso aos serviços.

A organização do sistema de saúde é o espelho da sociedade onde vivemos e este tem como objectivo assegurar a liberdade, a igualdade e a equidade na acessibilidade e utilização dos serviços a todos os cidadãos em função da sua necessidade de cuidados, de modo que a proteção da saúde seja um investimento social e que responda às expectativas legítimas da população. Com vista à análise da dimensão do acesso aos serviços de saúde por parte dos migrantes recorremos ao modelo teórico do acesso proposto por Aday \& Andersen (1974), e ao enquadramento proposto por Mladovsky (2009) para analisar as políticas de saúde migratórias.

Cada um destes modelos integra um conjunto de indicadores de medida, à luz dos quais foram analisados os resultados dos artigos incluídos nesta revisão. Os indicadores sugeridos por Aday \& Andersen são as políticas de saúde, as características do sistema de prestação de cuidados, as características da população em risco, a utilização dos serviços de saúde e a satisfação do consumidor. Por outro lado, Mladovsky considera a recolha de dados, os grupos populacionais atingidos, os problemas de saúde apontados, a parte do sistema 
de saúde afectado e a implementação. De acordo com a mesma autora "este processo pode ajudar os países a aprender com as experiências de outros e beneficiar da forma como cada política de saúde é desenhada em particular" (Mladovsky, 2009, p. 62).

Esta revisão tem por objectivo último sintetizar os efeitos da crise económica e das políticas de austeridade na saúde e no acesso aos cuidados de saúde da população migrante em Portugal, Espanha, Itália e Grécia, de forma a tornar visível as lacunas na evidência que ainda subsistem e, ao mesmo tempo, agregar e comparar a informação existente, de modo a contornar os desafios de saúde pública associados aos fluxos migratórios no Mediterrâneo atuais e passados, e mais particularmente aqueles que decorrem da crise e das políticas de austeridade que daí sucederam.

\section{Métodos}

Foi realizada uma revisão de literatura (Scoping Review) seguindo o protocolo proposto por Arksey \& O'Malley (2005). Procurou-se identificar publicações indexadas sobre os efeitos da crise económica e das políticas de austeridade na saúde da população migrante em países do sul da Europa, através de uma pesquisa eletrónica nas seguintes bases de dados: MEDLINE/ PubMed, Web of Science, EconLit e SciELO. Estas bases congregam artigos da área das ciências sociais e das ciências da saúde publicados em espanhol, português e inglês, razões pelas quais foram selecionadas.

A expressão de pesquisa foi desenhada tendo por base três filtros temáticos: a) Crise Económica e as suas Implicações; b) Saúde e Acesso aos Cuidados de Saúde; c) Populações Migrantes. Recorreu-se a uma combinação de termos de pesquisa adaptados (Kentikelenis et alii, 2015; Karanikolos et alii, 2016) que resultou na aplicação dos seguintes descritores: 'crisis', 'recession', 'downturn', 'austerity', 'economic crisis', 'fiscal crisis', 'financial crisis', 'economic recession', 'economic depression', 'economic insecurity', 'banking crisis', 'macroeconomic conditions', 'debt', 'unemployment', 'personnel downsizing', 'job loss', 'business cycle', 'health', 'health status', 'health care', 'health care access', 'health care availability', 'health care disparity', 'health care inequality', 'health care cost', 'migrant', 'immigrant', 'emigrant', 'asylum seeker', 'refugee', 'irregular', 'undocumented', 'foreign', 'citizen', 'nationalist', 'migration', 'immigration', 'emigration', 'marginalized' e 'vulnerable groups'. Optou-se por não se incluir na expressão de pesquisa os nomes dos quatro países em estudo de modo a evitar a perda de artigos em que não são mencionados nos títulos nem nos resumos mas que incluem dados sobre eles.

A busca eletrónica foi efetuada em Julho de 2016. Utilizou-se um filtro temporal com início em Janeiro de 2009, altura em que terão começado a 
ser publicados os primeiros estudos sobre os efeitos da crise económica que se iniciou em 2008. Os critérios de inclusão estabelecidos foram: i) artigos de investigação originais com dados empíricos; ii) que abordassem os impactos da crise económica e/ou políticas de austeridade na saúde ou acesso aos cuidados de saúde; iii) por parte de pelo menos um grupo migrante; iv) em Portugal, Espanha, Itália e/ou Grécia; v) escritos em línguas faladas pela equipa de pesquisa, incluindo o inglês, português, espanhol ou italiano. Definiu-se como população migrante todas as pessoas nascidas fora dos quatros países visados, e que aí residem, independentemente das causas da sua deslocação, do seu estatuto legal, e da duração da sua estadia, incluindo refugiados, requerentes a asilo, estudantes e imigrantes económicos (Crush, 2015).

A pesquisa resultou na identificação de 1.187 artigos, após exclusão de duplicados, que foram armazenados com recurso ao gestor de referências bibliográficas Mendeley.

Numa primeira fase, a seleção dos artigos foi realizada através da leitura dos títulos e resumos com recurso ao programa MS Office Access, sendo excluídos aqueles que claramente não cumpriam os requisitos de inclusão, restando 85 . Numa segunda fase, foram obtidos e analisados na íntegra os textos de todos os artigos potencialmente elegíveis sendo incluídos aqueles que contemplavam a proposta da presente revisão. Foram feitos dois pedidos de artigos ao próprio autor através do Researchgate, para os quais não se obteve resposta, aplicando-se a sua exclusão sob o critério de "indisponível". Os processos de seleção e avaliação foram realizados independentemente por dois investigadores, resolvendo-se as discrepâncias com recurso à análise e decisão por parte de um terceiro investigador. O processo de seleção resultou na inclusão de 18 artigos.

Os dados extraídos dos artigos incluídos foram inseridos numa tabela do MS Office Excel e agrupados de acordo com as seguintes características: autor, ano de publicação, país do estudo, método utilizado, população migrante visada, impactos no estado de saúde e impactos no acesso aos cuidados de saúde.

\section{Resultados}

De entre os 18 estudos que cumpriram com os critérios de inclusão na scoping review, a grande maioria foram realizados em Espanha ( $\mathrm{n}=15)$, seguindo-se a Itália $(n=2)$ e a Grécia $(n=1)$. Não foram identificados quaisquer estudos referentes a Portugal. Os estudos foram publicados entre 2013 e 2016 e adotaram abordagens metodológicas qualitativas $(\mathrm{n}=7)$, quantitativas $(\mathrm{n}=$ $10)$ e mistas $(n=1)$. As populações de pesquisa foram definidas em função da experiência laboral $(n=6)$, origem $(n=4)$, género $(n=2)$, doença $(n=2)$, estatuto legal $(n=1)$, idade $(n=1)$ e ausência de moradia $(n=1)$. Num dos estudos não é adiantada nenhuma especificidade da população migrante estudada. 
Os resultados obtidos demonstram o impacto negativo que estas reformas tiveram nos diferentes indicadores de saúde e nas populações mais vulneráveis, sendo agrupados com base em dois grandes temas: impactos no estado de saúde e impactos no acesso aos cuidados de saúde.

\section{Impacto no Estado de Saúde}

\section{Perceção Subjetiva do Estado de Saúde}

Os migrantes apresentam uma perceção subjetiva de saúde negativa. Está associada à degradação das condições laborais e da oferta de emprego durante a recessão, o que originou um impacto negativo na sua saúde mental (aumento do stress e depressão) e saúde física (dor generalizada) (Ronda et alii, 2016 ).

Para além destes fatores, a discriminação, o não reconhecimento de diplomas e a falta de apoio social têm também um impacto negativo na avaliação desta variável. Já a perceção de pertença a uma classe social baixa contribui para o aumento de fontes internas de stress emocional que resultam em comportamentos nocivos para a saúde (Sanchón-Macias et alii, 2016), sendo que os homens tendem a fazê-lo através de comportamentos de risco (alcoolismo e tabagismo) e as mulheres apresentam sintomatologias associadas como a depressão e a ansiedade.

\section{Saúde Ocupacional}

O presentismo, ou a presença no trabalho por parte de um trabalhador que se encontra doente, tem consequências nefastas para a saúde, tais como problemas respiratórios e doenças músculo-esqueléticas (Galon et alii, 2014), e encontra-se associado às más condições laborais, ao medo de ficar desempregado, a relações entre empregadores e empregados pautadas por exigências excessivas, pressões, ameaças ou abusos e à dificuldade em encontrar trabalhadores substitutos. Os imigrantes estiveram mais expostos a fatores psicossociais adversos no trabalho quando comparados com os trabalhadores espanhóis, apresentando uma maior prevalência de baixo controlo no trabalho, baixo apoio social, exigência física e insegurança elevadas (Torá et alii, 2015; Ronda et alii, 2016).

\section{Saúde Mental}

Muitos estudos referem consequências negativas para a saúde mental dos imigrantes como resultado da crise económica (Agudelo-Suárez et alii, 2013; Galon et alii, 2014; Gotsens et alii, 2015; Márquez, Urraza, 2016; Robert et alii, 2014, Ronda et alii, 2016), entre eles, o aumento de sintomas depressivos, ansiedade e stress (Galon et alii, 2014; Ronda et alii, 2016). Esses efeitos são mais notórios entre os imigrantes do sexo masculino que ficaram desempregados ou que trabalham em condições precárias e que têm encargos familiares. 
No que concerne ao consumo de medicamentos psicotrópicos, foi identificado um claro aumento do uso destes medicamentos em Espanha no período compreendido entre 2006 e 2012, tanto entre nativos como imigrantes, mas esse aumento foi muito mais expressivo no seio da população imigrante, e particularmente entre homens imigrantes que ficaram desempregados durante o período da crise (Gotsens et alii, 2015).

\section{Doenças Transmissíveis}

A falta de investimento em políticas de integração de imigrantes na Grécia e as políticas de austeridade, como a restrição do acesso aos serviços de saúde dos imigrantes em situação irregular, implementadas em Espanha poderão estar a contribuir para o aumento de doenças transmissíveis nesses países.

Um estudo que avaliou a incidência e a adesão terapêutica na tuberculose na Grécia antes e após o início da crise económica - 2007, 2010 e 2011 - concluiu que o número de pessoas infetadas com esta doença aumentou durante os anos da crise, tanto no caso dos gregos como no caso dos imigrantes (Sotiropoulou et alii, 2015). Esse aumento foi mais saliente na população migrante do que na população nativa entre o ano 2007 e o ano 2010. A maioria dos imigrantes que contraíram a doença residia na mesma área geográfica e foram infetados logo após a chegada ao país de acolhimento.

Segundo os autores, a segregação de imigrantes recém-chegados tem consequências nefastas para a sua saúde, sendo as políticas nacionais ainda insuficientes para colmatar este problema.

Muito embora não tenham sido identificados estudos sobre a incidência e prevalência de doenças transmissíveis nos outros países visados por esta revisão, existem estudos realizados em Espanha que apontam para o impacto negativo da aplicação do Real Decreto-lei 16/2012 no acesso dos imigrantes com doenças infeciosas e em situação irregular aos serviços de saúde, o que tem implicações negativas em termos da continuidade dos tratamentos e do seguimento, podendo ainda colocar em causa o controlo das doenças transmissíveis (Castano et alii, 2016).

\section{Doenças Não Transmissíveis}

A degradação das condições de trabalho associada à crise económica teve implicações negativas na saúde dos trabalhadores imigrantes traduzindo-se no aumento de doenças não transmissíveis como as lesões músculo-esqueléticas a nível lombar, as doenças respiratórias (tais como a asma) e as doenças somáticas (tais como dores musculares, cefaleias ou desconforto gástrico) (Galon et alii, 2014; Ronda et alii, 2016). Os imigrantes referiram ainda o uso de medicação para dormir como forma de diminuir o desconforto causado por estas sintomatologias (Ronda et alii, 2016). 


\section{Saúde Infantil}

As famílias de crianças imigrantes que têm um baixo nível educacional, elevadas taxas de desemprego e um elevado número de membros no agregado familiar tendem a enfrentar condições de precariedade económica que afetam negativamente a sua capacidade de comprar alimentos ou medicamentos prescritos e de imunizar as crianças quando as vacinas não são comparticipadas pelo estado (Martín Martín et alii, 2016).

\section{Impacto no Acesso aos Cuidados de Saúde}

\section{Modelo de Aday \& Andersen (1974)}

\section{Políticas de Saúde}

Num estudo realizado em Itália foi detetada a relação entre a pouca acessibilidade aos serviços de saúde por migrantes sem visto de residência (Cornaggia et alii, 2016). No entanto, outros autores realçam a existência de programas de boas práticas na identificação e inserção de indivíduos com desordens mentais, a colaboração e coordenação entre diferentes serviços, ou também, a disseminação de informação sobre serviços/prestadores de cuidados aos grupos marginalizados (Vittadini et alii, 2014).

Pode afirmar-se que uma melhoria do acesso aos serviços de saúde é um objetivo importante para a saúde pública (Aday, Andersen, 1974).

No entanto, em Espanha, devido ao RDL 16/2012 deparamo-nos com um sistema de saúde não universal, o que traduz uma desigualdade no seu acesso (Gotsens et alii, 2015), levando a que a duração dos processos de incorporação social aumente, devido aos cortes em certos benefícios sociais nos migrantes sem-abrigo, entre outros (Márquez, Urraza, 2016).

\section{Características do Sistema de Prestação de Cuidados}

Neste ponto são realçadas a insuficiente disponibilidade de recursos devido aos cortes, a redução do pessoal com o consequente aumento das listas de espera, bem como a necessidade de apoio por parte das ONG's na prestação de cuidados (Briones-Vozmediano et alii, 2014), ou ainda, o constante adiamento de cirurgias (Galon et alii, 2014).

Na mesma linha e relativo à organização, um estudo realizado na Grécia indica a diminuição gradual da duração média do tratamento da tuberculose, sendo que após o diagnóstico os migrantes são referenciados para um seguimento periódico com custos (Sotiropoulou et alii, 2015). Em Espanha, nas regiões da Andaluzia e Catalunha, as elevadas distâncias geográficas e os horários dos serviços de saúde são uma barreira ao acesso e utilização, já que não estão adaptadas às precárias condições de trabalho dos migrantes, além da diminuição da qualidade existente (Vázquez et alii, 2016). 


\section{Características da População em Risco}

Foi atribuída aos migrantes e às suas famílias em Espanha a componente de não-facilidade na perda do visto de residência e de trabalho, vulnerabilizando mais ainda uma população que vê diminuídos os ingressos financeiros e possíveis seguros de saúde associados (Gotsens et alii, 2015). Neste item, um estudo vem acentuar os custos como condicionante de acesso, ao concluir que quando os medicamentos prescritos e a vacina para o rotavírus não são financiados, estes não são comprados, expondo os indivíduos a graves consequências de saúde (Martín Martín et alii, 2016).

\section{Utilização dos Serviços de Saúde}

Na Grécia os migrantes com tuberculose ingerem o medicamento de forma controlada e observada pelos profissionais de saúde. O propósito deste tratamento baseia-se num maior controlo, já que o tempo de residência média no país ou nos locais onde residem (considerados guetos) são propícios à infecção imediata (Sotiropoulou et alii, 2015).

Os migrantes infectados com VIH, em situação irregular em Espanha, apresentam uma descontinuidade nos tratamentos (Pérez-Molina, Pulido, 2015). Eles não são atendidos ou veem o seu tratamento ser interrompido pela exigência de pagamento ou a não dispensa de medicamentos, facilitando a possível aparição de resistências aos anti-retrovirais ou o uso de tratamentos mais complexos e de maior custo no futuro. Simultaneamente é descrito que a assistência dada pelas ONG's fica comprometida devido aos cortes no próprio orçamento.

Quanto à utilização do médico de família, houve um aumento da prevalência entre 2006 e 2012. As consultas com especialistas mantiveram os mesmos níveis, bem como, o uso dos serviços de urgência (à excepção dos partos), apesar de este diminuir entre os anos de estudo. As mulheres migrantes utilizam com mais frequência o médico de clínica geral, mas o facto de apresentar um seguro privado de saúde está significativamente associado a uma maior utilização de especialistas. Já a prevalência de hospitalizações é superior nas classes sociais mais pobres. Os migrantes que trabalham têm uma taxa de utilização dos serviços superior aos desempregados (GarciaSubirats et alii, 2014).

\section{Satisfação do Consumidor}

Este aspeto refere-se ao grau de satisfação sentido pelos utilizadores acerca da quantidade e qualidade dos serviços de saúde recebidos, e abarca uma serie de percepções subjectivas, tais como, a conveniência, os custos, a coordenação, a cortesia, a informação e a qualidade. 
Neste ponto, há autores que referem que a informação dada aos migrantes sobre os seus problemas de saúde é pobre ou inexistente (Pérez-Molina, Pulido, 2015; Sotiropoulou et alii, 2015), ou ainda que os mecanismos de informação acerca das consultas, tais como o correio e o telefone, são inadequados devido às frequentes mudanças de endereço ou do número de telefone (Vázquez et alii, 2016). Para corroborar estes dados, há resultados descritos que concluem que migrantes que experienciaram uma doença infeciosa não têm informações quanto aos seus direitos de acesso aos serviços sanitários, nem sobre quem lhes pode prestar cuidados, levando a reacções de desapontamento, falta de confiança no sistema, medo ou mesmo abandono (Castano et alii, 2016).

\section{Modelo de Mladovsky (2009)}

\section{Problemas de Saúde Apontados}

Apesar do impacto negativo sentido pelos migrantes no acesso aos serviços de saúde em Espanha, as necessidades em saúde não atendidas diminuíram de 2006 para 2012, tais como os tempos de espera, a não obtenção de consulta ou a distância. No entanto, estes autores realçam que a equidade no acesso não garante igual equidade em cuidados de qualidade (Garcia-Subirats et alii, 2014).

\section{Parte do Sistema de Saúde Afectado}

Algumas das principais dificuldades manifestadas pelos migrantes para aceder ao sistema sanitário em Espanha são as barreiras no idioma (BrionesVozmediano et alii, 2014) ou a complexidade administrativa causada pela imensa burocracia e despesas associadas (Pérez-Molina, Pulido, 2015).

As mulheres latino-americanas, e apesar do mesmo idioma, podem deparar-se com um choque cultural e com horários incompatíveis para as consultas, em muitos casos sobreponíveis ao seu horário de trabalho (SanchónMacias et alii, 2016).

$\mathrm{Na}$ Catalunha os migrantes sem cartão de saúde têm de pagar taxas para consultas e tratamentos, o que inviabiliza a eficácia e favorece as morbilidades em saúde. Uma das formas de aceder aos serviços é usar os cartões de saúde pertencentes a outras pessoas (Vázquez et alii, 2016). Os mesmos autores realçam que as atitudes discriminatórias dos profissionais de saúde ou as diferenças de género e crenças são outras dificuldades encontradas.

\section{Implementação}

O desenvolvimento e implementação de políticas migratórias de saúde é um desafio, considerando a sua natureza política e contestação pública. Esta foi uma das conclusões em relação à não prioridade governamental de alterar 
políticas perante atitudes de violência doméstica contra mulheres imigrantes em Espanha (Briones-Vozmediano et alii, 2014).

Considerando que as diferentes Comunidades Autónomas espanholas seguem rigidamente o RDL 16/2012 e outras aplicam a sua própria legislação, encontramos diferenças no acesso aos serviços de saúde (Pérez-Molina, Pulido, 2015). Assim, no mesmo país, temos um conceito de saúde heterogéneo, inequitativo e díspar na sua universalidade, já que distingue a situação de regularidade de uma pessoa, e dentro da irregularidade, o acesso de certos grupos, como as grávidas, menores e urgências, ou se está registado com carência económica na cidade onde vive.

$\mathrm{Na}$ Catalunha, por exemplo, sendo esta também uma comunidade com sensibilidade para com a questão migratória, são identificadas desigualdades após a implementação do RDL 16/2012 através da limitação do acesso aos serviços de saúde em função da empregabilidade e do aumento do uso dos serviços de urgência (Castano et alii, 2016). Os resultados podem ser prejudiciais a médio/longo prazo a nível epidemiológico, já que se pode pôr em risco o tratamento de doenças infeciosas e economicamente ser muito custoso o agravamento de doenças crónicas associadas. Já em Madrid, num estudo que integra o depoimento de profissionais de saúde, todos eles responderam às restrições do RDL 16/2012 continuando a providenciar cuidados de saúde às populações excluídas, nomeadamente aos migrantes sem documentação. Os argumentos utilizados são de âmbito epidemiológico e economicista (Heras-Mosteiro, Sanz-Barbero, Otero-Garcia, 2016).

\section{Discussão}

Esta revisão de literatura teve como foco a análise das evidências disponíveis sobre os efeitos da crise económica de 2008 e das consequentes políticas de austeridade no estado de saúde e no acesso aos cuidados de saúde da população migrante em quatro países do sul da Europa.

Os resultados obtidos demonstram o impacto negativo que estas reformas tiveram nos diferentes indicadores de saúde e nas populações mais vulneráveis. A maioria dos estudos reportam dados de Espanha, e apenas uma pequena parte é relativa ao contexto italiano e grego. Não existem resultados acerca de Portugal, apesar da existência de muita literatura cinzenta, e mesmo quando a crise teve um severo impacto em todas estas economias. No entanto, aparentemente parece existir um padrão comum ao longo destes países, devido à partilha de certas semelhanças económicas, geográficas e sociodemográficas.

O impacto sobre os indicadores de saúde varia de acordo com o contexto nacional e com as respostas políticas dadas num momento de crise 
económica. Em Espanha, por exemplo, a mudança de paradigmas fez ceder um sistema de saúde universalista para adotar um modelo baseado em certos condicionantes, sendo o acesso limitado a determinados grupos populacionais mais vulneráveis, como os imigrantes indocumentados.

Os 18 estudos analisados exploram um conjunto de 14 indicadores de saúde na população migrante. Independentemente das diferenças de género, idade ou país de origem são encontradas incidências e prevalências nas disparidades e desigualdades no acesso aos serviços e no estado de saúde devido à exposição de certos determinantes de saúde, comportamentos e estilos de vida. Todavia, há que considerar ainda a existência de desigualdades estruturais e que o corte nos orçamentos para a saúde degradou as respostas da saúde pública e do seu ambiente legal.

As elevadas taxas de desemprego ou as dificuldades de inserção no mercado laboral, bem como, em caso de empregabilidade as degradadas condições como a redução salarial conduzem a piores condições de vida, habitacionais, alimentares ou mesmo à pobreza. Se aliarmos isto aos cortes na saúde pública e ao encerramento de programas preventivos podemos fomentar o aparecimento de doenças infeciosas. No entanto, estes valores podem não transparecer a realidade, dada a subnotificação de certos grupos populacionais sem acesso a qualquer diagnóstico de saúde.

A situação irregular de residência, a falta de suporte social e familiar ou as barreiras culturais são outros desafios em saúde que podem levar à marginalização e exclusão social, bem como, ao consumo de produtos aditivos, com consequências na saúde mental. É extremamente necessária a proteção da sanidade mental dos grupos mais vulneráveis.

A proteção laboral é outro aspeto relevante, já que podem estar em causa doenças ou lesões, que possam vir a impedir o migrante de trabalhar ou resultar em situações de maior gasto dos serviços de saúde.

Por último, a adaptação dos serviços de saúde às populações imigrantes, bem como, a expansão das competências culturais pelos profissionais de saúde torna-se imprescindível, de maneira a que o migrante possa aceder aos serviços de saúde e se interligue com uma equipa multidisciplinar na pro-actividade da sua prestação de cuidados.

O desenvolvimento e implementação de medidas é potencialmente um desafio, considerando a contestação pública e a natureza política relacionada com as migrações. As diferenças entre países e as suas próprias necessidades determinam a gama de intervenções em saúde aplicadas, que até ao momento pouco alcance obtiveram na equidade e igualdade no acesso ao sistema de saúde por parte dos migrantes, já que "as barreiras para ao acesso 
não são apenas financeiras, mas também psicológicas, informativas, sociais, organizacionais, espaciais, temporais, e por aí" (Aday, Andersen, 1974, p. 219).

Por outro lado, os países do sul da Europa dada a sua localização como porta de entrada e de trânsito num continente tão apetecido a norte, desenvolveram um intensivo controlo fronteiriço na tentativa de bloquear as intenções migratórias. No entanto, no reverso da situação são tomadas atitudes displicentes, no próprio território, ao obliterar os direitos humanos de cada migrante que aguarda por uma decisão legal da sua situação, deixando-os à mercê num limbo perigoso.

O que este estudo traz de novo em relação aos já realizados é a síntese de um conjunto de resultados de saúde, em três países mediterrânicos, sobre o estado de saúde e o acesso aos serviços de saúde por parte de populações imigrantes. Isto facilitará e servirá de apoio à investigação sobre os impactos na saúde produzidos pela crise económica e políticas de austeridade aplicadas.

Como limitações temos os critérios de inclusão e bases de dados que podem ter levado à não inserção de alguns documentos relevantes ou de documentos pertencentes à literatura cinzenta, não avaliados pelos pares científicos, mas que podem enriquecer com as seus resultados e conclusões. Também é de considerar a falta de estudos que abordam o contexto português, sendo que o objetivo inicial seria alcançar resultados em quatro países, bem como, a diminuta contribuição da Itália e Grécia. Outro aspeto são os dados obtidos sobre os resultados em saúde, que serão sempre revelados com "atraso" já que necessitam de tempo para evidenciarem as suas consequências na morbilidade ou mortalidade das populações, ao contrário dos dados económicos que oferecem resultados mais imediatos.

Relacionado com isto, podemos indicar também as falhas de conhecimento, a melhorar, derivadas da imprevisível mutação do contexto económico ou ainda da volatilidade do ambiente político dos países receptores. Torna-se por isso necessário continuar a acompanhar detalhadamente esta situação e a descrever a evolução verificada.

Estas limitações apontam uma clara necessidade de maior pesquisa e investigação sobre a saúde dos migrantes e sobre os efeitos das crises económicas, através de um leque variado de estudos sobre os países abordados.

\section{Conclusão}

Através da análise do estado de saúde e do acesso aos serviços sanitários em três países do sul da Europa, esta scoping review oferece uma visão de como a aplicação de políticas de austeridade na saúde pode ter um impacto negativo e sequelas adversas nas populações migrantes, nomeadamente quando os países atravessam uma grave crise económica. 
Se por um lado, os governos nacionais têm a obrigação de garantir uma adequada cobertura de saúde a toda a população, por outro lado, a crise económica conduziu a alterações nas políticas destes países, que colocaram em causa a igualdade nos direitos e no acesso a recursos, nomeadamente dos grupos populacionais mais vulneráveis como os migrantes.

A colheita de dados empíricos sobre o acesso aos serviços de saúde permite diferenciar a disponibilidade e a utilização dos serviços pelos diferentes sub-grupos das populações mais pobres, com o objetivo final de comparar a evidência nestes países.

Os resultados deste estudo oferecem aos países em questão e às organizações envolvidas nas políticas migratórias a oportunidade de reconhecer as suas semelhanças e divergências, mas acima de tudo, de partilharem oportunidades de aprendizagem e refletirem sobre a necessidade de alteração das políticas de saúde dirigidas a estas populações, com vista à obtenção de uma maior equidade em saúde.

Recomenda-se a realização de mais investigação que permita colmatar as lacunas existentes na evidência dos impactos da crise no estado de saúde e no acesso aos serviços de saúde das populações, especialmente em países como Portugal, Itália e Grécia. Sugere-se ainda o fortalecimento da acessibilidade dos cuidados de saúde, nomeadamente através do restabelecimento da universalidade do acesso, e de uma aposta na prevenção da doença e promoção da saúde, já que a longo prazo estas medidas tendem a favorecer a equidade em saúde e a revelar-se mais económicas. É importante, também, proteger legislativamente os migrantes num ambiente favorável, e estabelecer programas de acção comunitária que reforcem e desenvolvam as suas aptidões e os recursos individuais.

Desta forma, conclui-se que as perspectivas futuras são um desafio para os sistemas socio-sanitários em sociedades diversas, mas simultaneamente devem ser encaradas de forma encorajadora e audaz, já que as alterações nas políticas de saúde podem contribuir para a criação de sociedades mais fortes, resilientes e empoderadas.

\section{Bibliografia}

ADAY, Lu; ANDERSEN, Ronald. A Framework for the Study of Access to Medical Care. Health Service Research, p. 208-220, 1974.

AGUDELO-SUÁREZ, Andrés; RONDA, Elena; VÁZQUEZ-NAVARRETE, María; GARCÍA, Ana; MARTíNEZ, José; BENAVIDES, Fernando. Impact of economic crisis on mental health of migrant workers: what happened with migrants who came to Spain to work? International Journal of Public Health, v. 58, n. 4, p. 627-631, 2013. ARKSEY, Hilary; O'MALLEY, Lisa. Scoping studies: towards a methodological framework. International Journal of Social Research Methodology, v. 8, n. 1, p. 19-32, 2005. 
Boletín Oficial del Estado. Real Decreto-ley 16/2012, de 20 de abril, de medidas urgentes para garantizar la sostenibilidad del Sistema Nacional de Salud y mejorar la calidad y seguridad de sus prestaciones. 2012. Disponível em: <https://www. boe.es/boe/dias/2012/04/24/pdfs/BOE-A-2012-5403.pdf>.

BRIONES-VOZMEDIANO, Erica; AGUDELO-SUAREZ, Andrés; GOICOLEA, Isabel; VIVES-CASES, Carmen. Economic crisis, immigrant women and changing availability of intimate partner violence services: a qualitative study of professionals' perceptions in Spain. International Journal for Equity in Health, v. 13, n. 1, p. 79, 2014.

CASTANO, Jenny; OSPINA, Jesús; CAYLÀ, Joan; GREER, Scott. Restricting Access to Health Care to Immigrants in Barcelona. International Journal of Health Services, v. 46, n. 2, p. 241-261, 2016.

CORNAGGIA, Cesare; BEGHI, Massimiliano; MEZZANZANICA, Mario; RONZONI, Gloria; VITTADINI, Giorgio; MAFFENINI, Walter. Psychotropic Drug Consumption and Employment Status in Time of Economic Crisis (2007-2011). Psychiatric Quarterly, v. 88, n. 2, p. 371-384, 2016.

CRUSH, Jonathan. Global Migration. In: International Encyclopedia of the Social \& Behavioral Sciences. Kidlington: Elsevier, 2015, p. 169-173.

GALON, Tanyse; BRIONES-VOZMEDIANO, Erica; AGUDELO-SUÁREZ, Andrés; FELT, Emily; BENAVIDES, Fernando; RONDA, Elena. Understanding sickness presenteeism through the experience of immigrant workers in a context of economic crisis. American Journal of Industrial Medicine, v. 57, n. 8, p. 950-959, 2014.

GARCÍA-RAMÍrEZ, Manuel; BALCÁZAR, Fabricio; DE FREITAS, Cláudia. Community psychology contributions to the study of social inequalities, wellbeing and social justice. Psychosocial Intervention, v. 23, n. 2, p. 79-81, 2014.

GARCIA-SUBIRATS, Irene; VARGAS, Ingrid; SANZ, Belén; MALMUSI, Davide; RONDA, Elena; BALLESTA, Mónica; Vázquez, María. Changes in Access to Health Services of the Immigrant and Native-Born Population in Spain in the Context of Economic Crisis. International Journal of Environmental Research and Public Health, v. 11, n. 10, p. 10182-10201, 2014.

GOTSENS, Mercè; MALMUSI, Davide; VILLARROEL, Nazmy; VIVES-CASES, Carmen; GARCIA-SUBIRATS, Irene; HERNANDO, Cristina; BORRELL, Carme. Health inequality between immigrants and natives in Spain: the loss of the healthy immigrant effect in times of economic crisis. European Journal of Public Health, v. 25, n. 6, p. 923-929, 2015.

HERAS-MOSTEIRO, Julio; SANZ-BARBERO, Belén; OTERO-GARCIA, Laura. Health Care Austerity Measures in Times of Crisis. International Journal of Health Services, v. 46, n. 2, p. 283-299, 2016.

INGLEBY, David; CHIMIENTI, Milena; HATZIPROKOPIOU, Panos; ORMOND, Meghann; DE FREITAS, Cláudia. The Role of Health in Integration. In FONSECA, Lucinda; MALHEIROS, Jorge (orgs.). Social integration and mobility: education, housing and health. IMISCOE Cluster B5 State of the art report. Lisboa: Centro de Estudos Geográficos, Universidade de Lisboa, 2005, p. 88-119. 
International Organization for Migration. IOM Response Plan for the Mediterranean and Beyond Addressing complex migration flows in the countries of origin, transit and destination. Vienna: October, 2015.

KARANIKOLOS, Marina; HEINO, Pia; MCKEE, Martin; STUCKLER, David; LEGIDOQUIGLEY, Helena. Effects of the Global Financial Crisis on Health in High-Income Oecd Countries: A Narrative Review. International Journal of Health Services: Planning, Administration, Evaluation, v. 46, n. 2, p. 208-240, 2016.

KENTIKELENIS, Alexander; KARANIKOLOS, Marina; WILLIAMS, Gemma; MLADOVSKY, Philipa; KING, Lawrence; PHARRIS, Anastasia; ... STUCKLER, David. How do economic crises affect migrants' risk of infectious disease? A systematic-narrative review. European Journal of Public Health, v. 25, n. 6, p. 937944, 2015.

LEGIDO-QUIGLEY, Helena; KARANIKOLOS, Marina; HERNANDEZ-PLAZA, Sonia; DE FREITAS, Cláudia; BERNARDO, Luís; PADILLA, Beatriz; MACHADO, Rita Sá; DIAZ-ORDAZ, Karla; STUCKLER, David; MCKEE, Martin. Effects of the financial crisis and Troika austerity measures on health and health care access in Portugal. Health Policy, v. 120, n. 7, p. 833-839, 2016.

MALMUSI, Davide. Immigrants' health and health inequality by type of integration policies in European countries. European Journal of Public Health, v. 25, n. 2, p. 293-299, 2015.

MALMUSI, Davide; ORTIZ-BARREDA, Gaby. Desigualdades sociales en salud en poblaciones inmigradas en España: revisión de la literatura. Revista Española de Salud Pública, v. 88, n. 6, p. 687-701, 2014.

MÁRQUEZ, Gorka; URRAZA, Xabier. The impact of the crisis on homelessness in Spain: social intervention in a context of greater need and fewer resources. European Journal of Social Work, v. 19, n. 1, p. 31-45, 2016.

MARTÍN MARTÍN, Raquel; SÁNCHEZ BAYLE, Marciano; GANCEDO GARCÍA, Carmen; TERUEL DE FRANCISCO, Ma; COULLAUT LÓPEZ, Ana. Las familias de la crisis en las consultas pediátricas de Atención Primaria: estudio descriptivo observacional. Anales de Pediatría, v. 84, n. 4, p. 189-194, 2016.

MLADOVSKY, Philipa. A framework for analysing migrant health policies in Europe. Health Policy, v. 93, n. 1, p. 55-63, 2009.

Organización Internacional para las Migraciones. Migración internacional, salud y derechos humanos. Ginebra, 2013. Disponível em: <http://www.ohchr.org/ Documents/Issues/Migration/WHO_IOM_UNOHCHRPublication_sp.pdf $>$.

PÉREZ-MOLINA, José; PULIDO, Federico. ¿Cómo está afectando la aplicación del nuevo marco legal sanitario a la asistencia de los inmigrantes infectados por el VIH en situación irregular en España? Enfermedades Infecciosas Y Microbiología Clínica, v. 33, n. 7, p. 437-445, 2015.

ROBERT, Gemma; MARTINEZ, José; GARCIA, Ana; BENAVIDES, Fernando; RONDA, Elena. From the boom to the crisis: changes in employment conditions of immigrants in Spain and their effects on mental health. European Journal of Public Health, v. 24, n. 3, p. 404-409, 2014. 
RONDA, Elena; BRIONES-VOZMEDIANO, Erica; GALON, Tanyse; GARCÍA, Ana; BENAVIDES, Fernando; AGUDELO- SUÁREZ, Andrés. A qualitative exploration of the impact of the economic recession in Spain on working, living and health conditions: reflections based on immigrant workers' experiences. Health Expectations, v. 19, n. 2, p. 416-426, 2016.

SANCHÓN-MACIAS, María; BOVER-BOVER, Andreu; PRIETO-SALCEDA, Dolores; PAZ-ZULUETA, María; TORRES, Blanca; GASTALDO, Denise. Determinants of Subjective Social Status and Health Among Latin American Women Immigrants in Spain: A Qualitative Approach. Journal of Immigrant and Minority Health, v. 18, n. 2, p. 436-441, 2016.

SOTIROPOULOU, Penelope; GOURGOULIANIS, Konstantinos; KONSTANTINOU, Konstantinos; PETINAKI, Efthimia; ROUPA, Zoe. Retrospective Study of Measuring Tuberculosis Therapy Compliance: Greece as a Host Country for Vulnerable Populations Before and During the Financial Crisis. Materia Socio Medica, v. 27, n. 5, p. 328-332, 2015.

TORÁ, Isabel; MARTíNEZ, José; BENAVIDES, Fernando; LEVEQUE, Katia; RONDA, Elena. Effect of economic recession on psychosocial working conditions by workers' nationality. International Journal of Occupational and Environmental Health, v. 21, n. 4, p. 328-332, 2015.

VÁZQUEZ, María-Luisa; VARGAS, Ingrid; JARAMILLO, Daniel; PORTHÉ, Victoria; LÓPEZ-FERNÁNDEZ, Luis; VARGAS, Hernán; BOSCH, Lola; HERNÁNDEZ, Silvia; ZAROLA, Ainhoa. Was access to health care easy for immigrants in Spain? The perspectives of health personnel in Catalonia and Andalusia. Health Policy, v. 120, n. 4, p. 396-405, 2016.

VITTADINI, Giorgio; BEGHI, Massimiliano; MEZZANZANICA, Mario; RONZONI, Gloria; CORNAGGIA, Cesare. Use of psychotropic drugs in Lombardy in time of economic crisis (2007-2011): A population-based study of adult employees. Psychiatry Research, v. 220, n. 1-2, p. 615-622, 2014.

Recebido para publicação em 13.04.2018

Aceito para publicação em 11.09.2018

Received for publication in April 13 ${ }^{\text {th }}, 2018$ Accepted for publication in September $11^{\text {th }}, 2018$

ISSN impresso 1980-8585

ISSN eletrônico 2237-9843

http://dx.doi.org/10.1590/1980-85852503880005411 